\title{
Existence of multiple positive solutions to integral boundary value systems with boundary multiparameters
}

Eunkyung Ko ${ }^{1}$ and Eun Kyoung Lee $2^{2^{*}}$

"Correspondence:

eklee@pusan.ac.kr

${ }^{2}$ Department of Mathematics

Education, Pusan National

University, Busan, South Korea

Full list of author information is

available at the end of the article

\begin{abstract}
We establish the existence, nonexistence, and multiplicity of positive solutions to semilinear elliptic systems with integral boundary conditions when positive multiparameters vary on the boundary. We prove the results by using sub- and supersolution argument and fixed point index theory.
\end{abstract}

MSC: $35 J 55 ; 35 J 25 ; 34 \mathrm{~B} 08 ; 34 \mathrm{~B} 10$

Keywords: Coupled integral boundary system; Boundary multiparameters; Multiple positive solutions; Fixed point index; Sub- and supersolutions

\section{Introduction}

In this paper, we study the existence of multiple positive radial solutions to the following nonlocal boundary value system with boundary parameters on an exterior domain:

$$
\begin{cases}\Delta u+K_{1}(|x|) f_{1}(u(x), v(x))=0, & x \in \Omega_{e}, \\ \Delta v+K_{2}(|x|) f_{2}(u(x), v(x))=0, & x \in \Omega_{e}, \\ u(x) \rightarrow 0, \quad v(x) \rightarrow 0 & \text { as }|x| \rightarrow \infty, \\ u(x)-\int_{\Omega_{e}} l_{1}(|y|) v(y) d y=\lambda & \text { if }|x|=r_{0}, \\ v(x)-\int_{\Omega_{e}} l_{2}(|y|) u(y) d y=\mu & \text { if }|x|=r_{0},\end{cases}
$$

where $\Omega_{e}=\left\{x \in(-\infty, \infty)^{N}:|x| \geq r_{0}\right.$ for $\left.r_{0}>0, N \geq 3\right\}, \lambda$ and $\mu$ are positive parameters, $K_{i} \in C\left(\left(r_{0}, \infty\right),(0, \infty)\right)$ is such that $\int_{r_{0}}^{\infty} r^{\nu} K_{i}(r) d r<\infty$ for some $v>1, f_{i} \in C\left([0, \infty)^{2},[0, \infty)\right)$, and $l_{i} \in L^{1}\left(\left(r_{0}, \infty\right)\right)$ are nonnegative functions satisfying $0<w_{N} r_{0}^{N-2} \int_{r_{0}}^{\infty} r l_{i}(r) d r<1$ for $i=1,2$, where $w_{N}$ is the surface area of the unit sphere in $(-\infty, \infty)^{N}$.

Differential equations with integral boundary conditions arise in various areas of applied mathematics and physics like heat conduction, chemical engineering, underground water flow, and thermo-elasticity and plasma phenomena. For integral boundary value problems, we refer to $[1,5,6,8,10-13]$, and the references therein.

(c) The Author(s) 2018. This article is distributed under the terms of the Creative Commons Attribution 4.0 International License (http://creativecommons.org/licenses/by/4.0/), which permits unrestricted use, distribution, and reproduction in any medium, provided you give appropriate credit to the original author(s) and the source, provide a link to the Creative Commons license, and indicate if changes were made. 
Note that the change of variables $r=|x|$ and $t=\left(\frac{r}{r_{0}}\right)^{2-N}$ transforms (1) into

$$
\begin{cases}u^{\prime \prime}(t)+a_{1}(t) f_{1}(u(t), v(t))=0, & t \in(0,1) \\ v^{\prime \prime}(t)+a_{2}(t) f_{2}(u(t), v(t))=0, \quad t \in(0,1) \\ u(0)=0=v(0) \\ u(1)-\int_{0}^{1} g_{1}(s) v(s) d s=\lambda, \\ v(1)-\int_{0}^{1} g_{2}(s) u(s) d s=\mu\end{cases}
$$

with

$$
\begin{aligned}
& a_{i}(t)=\left(\frac{1}{N-2}\right)^{2} r_{0}^{2} t^{\frac{-2(N-1)}{N-2}} K_{i}\left(r_{0} t \frac{-1}{N-2}\right), \\
& g_{i}(t)=w_{N}\left(\frac{1}{N-2}\right) r_{0}^{N} t^{\frac{-2(N-1)}{N-2}} l_{i}\left(r_{0} t^{\frac{-1}{N-2}}\right),
\end{aligned}
$$

where $a_{i} \in C((0,1),[0, \infty))$ are such that $\int_{0}^{1} s^{\alpha}(1-s)^{\beta} a_{i}(s) d s<\infty$ for some $\alpha, \beta \in(0,1)$, and nonnegative functions $g_{i} \in L^{1}(0,1)$ are such that $0<\int_{0}^{1} s g_{i}(s) d s<1$ for $i=1,2$. We further assume that there exists a closed interval $J \subset(0,1)$ of positive measure such that $a_{i}(t)>0$ for all $t \in J$ and $i=1,2$. Note that the existence of positive solutions of system (2) guarantees the existence of positive radial solutions of (1). Hence, to investigate solutions of (1), we focus on system (2).

Such second-order ordinary differential systems with the coupled integral boundary conditions were considered in [2,3], and [4]. Most of those results are about the existence of a solution obtained mainly by using the fixed point theorem, the mixed monotone method, and the monotone iterative method. In [4], the authors investigated the existence and uniqueness of positive solutions by using the a priori estimate method and the maximum principle. To the best of our knowledge, the multiplicity of solutions of differential systems with coupled integral boundary conditions has not been treated. In this paper, we study the existence, nonexistence, and multiplicity of positive solutions to a nonlocal boundary value system with integral boundary conditions when two positive multiparameters vary on the boundary. We establish our results by sub- and supersolution arguments and fixed point index theory.

In this paper, we assume the following hypotheses on $f_{i}$ for $i=1,2$.

$(\mathrm{H}) f_{i}:[0, \infty) \times[0, \infty) \rightarrow[0, \infty)$ is continuous and increasing in $[0, \infty) \times[0, \infty)$ for $i=1,2$.

(H1) $f_{i, 0}:=\lim _{\|(u, v)\| \rightarrow 0} \frac{f_{i}(u, v)}{u+v}=0$ for $i=1,2$.

(H2) $f_{i, \infty}:=\lim _{\|(u, v)\| \rightarrow \infty} \frac{f_{i}(u, v)}{u+v}=\infty$ for $i=1,2$.

Here $\|(u, v)\|=\|u\|_{\infty}+\|v\|_{\infty}$. Now we state our main result.

Theorem 1.1 There exists a continuous curve $\Gamma$ separating $[0, \infty) \times[0, \infty) \backslash\{(0,0)\}$ into two disjoint sets $\Theta_{1}$ and $\Theta_{2}$, and there exists a subset $\Theta \subset \Theta_{1}$ such that (2) has at least two positive solutions for $(\lambda, \mu) \in \Theta$, at least one positive solution for $(\lambda, \mu) \in\left(\Theta_{1} \backslash \Theta\right) \cup \Gamma$, and no positive solution when $(\lambda, \mu) \in \Theta_{2}$. (See Figure 1.)

The structure of $f_{1}$ and $f_{2}$ near $(0,0)$ and near $\infty$, that is, conditions $(\mathrm{H} 1)$ and $(\mathrm{H} 2)$, plays an important role in the construction of sub- and supersolutions when the parameters $\lambda$ 


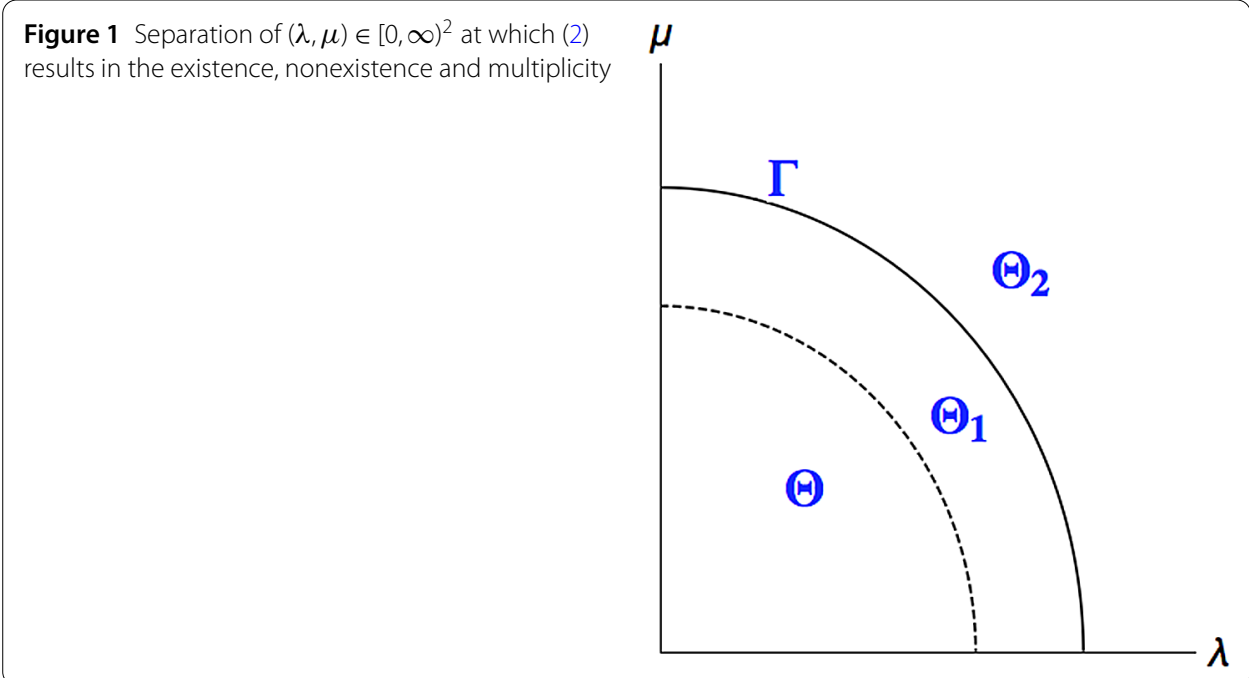

and $\mu$ vary. Hence system (2) in which two parameters $\lambda$ and $\mu$ involved with only the boundary data is difficult for construction of sub- and supersolutions at the certain value of $(\lambda, \mu)$. To overcome this issue, we manipulate (2) in such a way that the two parameters $\lambda$ and $\mu$ are related with $f_{1}$ and $f_{2}$ (see (4) in Sect. 2). Here we also emphasize that we consider the case where system (2) satisfies the condition $f_{i}(0,0)=0$ for $i=1,2$ and the boundary condition $u(0)=0=v(0)$ at the same time. This is a challenging case since these conditions do not allow us to construct a proper open set, which is very crucial for applying the fixed point index theory. Hence we perturb the boundary values of the manipulated system (4) so that the fixed point index argument works well (see (5) in Sect. 2).

The paper is organized as follows. In the next section, we present a system equivalent to (2) and introduce the sub- and supersolution theorem and fixed point index. Section 3 is devoted to the proof of the main result, Theorem 1.1. In Appendix, we prove the suband supersolution theorem for (2).

\section{Preliminaries}

Let $p_{\lambda, \mu}$ and $q_{\lambda, \mu}$ be the solutions, respectively, of the following problems:

$$
\left\{\begin{array}{l}
p^{\prime \prime}(t)=0, \\
p(0)=0, \\
p(1)-\int_{0}^{1} g_{1}(s) q(s) d s=\lambda,
\end{array} \quad t \in(0,1)\right.
$$

and

$$
\left\{\begin{array}{l}
q^{\prime \prime}(t)=0, \\
q(0)=0, \\
q(1)-\int_{0}^{1} g_{2}(s) p(s) d s=\mu .
\end{array} \quad t \in(0,1)\right.
$$

It is easy to see that

$$
p_{\lambda, \mu}(t)=\frac{1}{1-\sigma_{1} \sigma_{2}}\left(\lambda+\sigma_{1} \mu\right) t, \quad q_{\lambda, \mu}(t)=\frac{1}{1-\sigma_{1} \sigma_{2}}\left(\sigma_{2} \lambda+\mu\right) t, \quad t \in[0,1],
$$


where $\sigma_{1}=\int_{0}^{1} s g_{1}(s) d s$ and $\sigma_{2}=\int_{0}^{1} s g_{2}(s) d s$. We denote

$$
p_{\lambda, \mu}(t)=\left(\rho \lambda+\rho_{1} \mu\right) t, \quad q_{\lambda, \mu}(t)=\left(\rho_{2} \lambda+\rho \mu\right) t,
$$

where $\rho=\frac{1}{1-\sigma_{1} \sigma_{2}}, \rho_{1}=\frac{\sigma_{1}}{1-\sigma_{1} \sigma_{2}}$, and $\rho_{2}=\frac{\sigma_{2}}{1-\sigma_{1} \sigma_{2}}$. Note that $\rho, \rho_{1}$, and $\rho_{2}$ are positive constants.

Now if $\left(w_{1}, w_{2}\right)$ is a solution of the system

$$
\begin{cases}w_{1}^{\prime \prime}(t)+a_{1}(t) f_{1}\left(w_{1}(t)+p_{\lambda, \mu}(t), w_{2}(t)+q_{\lambda, \mu}(t)\right)=0, & t \in(0,1), \\ w_{2}^{\prime \prime}(t)+a_{2}(t) f_{2}\left(w_{1}(t)+p_{\lambda, \mu}(t), w_{2}(t)+q_{\lambda, \mu}(t)\right)=0, & t \in(0,1), \\ w_{1}(0)=0=w_{2}(0), & \\ w_{1}(1)-\int_{0}^{1} g_{1}(s) w_{2}(s) d s=0, & \\ w_{2}(1)-\int_{0}^{1} g_{2}(s) w_{1}(s) d s=0, & \end{cases}
$$

then $\left(w_{1}+p_{\lambda, \mu}, w_{2}+q_{\lambda, \mu}\right)$ is a solution of (2) since

$$
\left\{\begin{array}{l}
\left(w_{1}+p_{\lambda, \mu}\right)^{\prime \prime}(t)=w_{1}^{\prime \prime}(t)=-a_{1}(t) f_{1}\left(w_{1}+p_{\lambda, \mu}, w_{2}+q_{\lambda, \mu}\right), \quad t \in(0,1) \\
\left(w_{1}+p_{\lambda, \mu}\right)(0)=0 \\
\left(w_{1}+p_{\lambda, \mu}\right)(1)=\int_{0}^{1} g_{1}(s)\left(w_{2}(s)+q_{\lambda, \mu}(s)\right) d s+\lambda,
\end{array}\right.
$$

and, similarly, $w_{2}+q_{\lambda, \mu}$ satisfies (2). Hence, instead of studying system (2), we consider system (4) to get the existence and multiplicity of positive solutions of (2). However, for system (4) satisfying the conditions $f_{i}(0,0)=0$ and $w_{i}(0)=0, i=1,2$, there is an obstacle in using a fixed point index argument. To solve this issue, we employ the perturbed boundary condition.

For a while, let us consider the following problem with more general boundary conditions for $0 \leq \delta<\tilde{\delta}$ for sufficiently small $\tilde{\delta}$ ( $\tilde{\delta}$ will be chosen in Lemma 3.3 ):

$$
\begin{cases}w_{1}^{\prime \prime}(t)+a_{1}(t) f_{1}\left(w_{1}(t)+p_{\lambda, \mu}(t), w_{2}(t)+q_{\lambda, \mu}(t)\right)=0, & t \in(0,1), \\ w_{2}^{\prime \prime}(t)+a_{2}(t) f_{2}\left(w_{1}(t)+p_{\lambda, \mu}(t), w_{2}(t)+q_{\lambda, \mu}(t)\right)=0, & t \in(0,1), \\ w_{1}(0)=\delta=w_{2}(0), & \\ w_{1}(1)-\int_{0}^{1} g_{1}(s) w_{2}(s) d s=\delta, & \\ w_{2}(1)-\int_{0}^{1} g_{2}(s) w_{1}(s) d s=\delta . & \end{cases}
$$

We set up an operator equation for problem (5). We define

$$
\begin{aligned}
A_{\lambda, \mu, \delta}\left(w_{1}, w_{2}\right)(t):= & \delta\left[1+\left(\rho v_{1}+\rho_{1} v_{2}\right) t\right]+\int_{0}^{1} H_{2}(t, s) a_{1}(s) f_{1}\left(w_{1}+p_{\lambda, \mu}, w_{2}+q_{\lambda, \mu}\right) \\
& +K_{1}(t, s) a_{2}(s) f_{2}\left(w_{1}+p_{\lambda, \mu}, w_{2}+q_{\lambda, \mu}\right) d s \\
B_{\lambda, \mu, \delta}\left(w_{1}, w_{2}\right)(t):= & \delta\left[1+\left(\rho v_{2}+\rho_{2} v_{1}\right) t\right]+\int_{0}^{1} K_{2}(t, s) a_{1}(s) f_{1}\left(w_{1}+p_{\lambda, \mu}, w_{2}+q_{\lambda, \mu}\right) \\
& +H_{1}(t, s) a_{2}(s) f_{2}\left(w_{1}+p_{\lambda, \mu}, w_{2}+q_{\lambda, \mu}\right) d s
\end{aligned}
$$


where

$$
\begin{aligned}
& \left\{\begin{array}{l}
H_{1}(t, s)=G(t, s)+\rho_{2} t \int_{0}^{1} G(\tau, s) g_{1}(\tau) d \tau, \\
H_{2}(t, s)=G(t, s)+\rho_{1} t \int_{0}^{1} G(\tau, s) g_{2}(\tau) d \tau,
\end{array}\right. \\
& K_{1}(t, s)=\rho t \int_{0}^{1} G(\tau, s) g_{1}(\tau) d \tau, \quad K_{2}(t, s)=\rho t \int_{0}^{1} G(\tau, s) g_{2}(\tau) d \tau, \\
& G(t, s)= \begin{cases}s(1-t), & 0 \leq s \leq t \leq 1, \\
t(1-s), & 0 \leq t \leq s \leq 1,\end{cases}
\end{aligned}
$$

and

$$
v_{1}=\int_{0}^{1} g_{1}(s) d s, \quad v_{2}=\int_{0}^{1} g_{2}(s) d s .
$$

Then $\left(v_{1}(t), v_{2}(t)\right)=\left(A_{\lambda, \mu, \delta}\left(w_{1}, w_{2}\right)(t), B_{\lambda, \mu, \delta}\left(w_{1}, w_{2}\right)(t)\right)$ is a solution of

$$
\begin{cases}v_{1}^{\prime \prime}(t)+a_{1}(t) f_{1}\left(w_{1}(t)+p_{\lambda, \mu}(t), w_{2}(t)+q_{\lambda, \mu}(t)\right)=0, & t \in(0,1), \\ v_{2}^{\prime \prime}(t)+a_{2}(t) f_{2}\left(w_{1}(t)+p_{\lambda, \mu}(t), w_{2}(t)+q_{\lambda, \mu}(t)\right)=0, & t \in(0,1), \\ v_{1}(0)=\delta=v_{2}(0), & \\ v_{1}(1)-\int_{0}^{1} g_{1}(s) v_{2}(s) d s=\delta \\ v_{2}(1)-\int_{0}^{1} g_{2}(s) v_{1}(s) d s=\delta .\end{cases}
$$

Now we define

$$
T_{\lambda, \mu, \delta}\left(w_{1}, w_{2}\right)(t):=\left(A_{\lambda, \mu, \delta}\left(w_{1}, w_{2}\right)(t), B_{\lambda, \mu, \delta}\left(w_{1}, w_{2}\right)(t)\right) .
$$

Let us denote $X:=C([0,1],(-\infty, \infty)) \times C([0,1],(-\infty, \infty))$. Then $T_{\lambda, \mu, \delta}: X \rightarrow X$ is well defined on $X$, where $X$ is the usual Banach space with the norm $\|(u, v)\|=\|u\|_{\infty}+\|v\|_{\infty}$. Notice that problem (5) is equivalent to

$$
\left(w_{1}, w_{2}\right)=T_{\lambda, \mu, \delta}\left(w_{1}, w_{2}\right) \quad \text { on } X
$$

Let $\mathcal{P}=\{(u, v) \in X: u(t) \geq 0, v(t) \geq 0$ for all $t \in[0,1]\}$. Then $\mathcal{P}$ is a cone in $X$. It is clear that $T_{\lambda, \mu, \delta}(\mathcal{P}) \subset \mathcal{P}$ and $T_{\lambda, \mu, \delta}$ is completely continuous on $X$ by a standard argument.

Now, we introduce a theorem on sub- and supersolutions to system (5). First, we state the following definition of subsolution and supersolution of system (5).

Definition 2.1 We say that $\left(\psi_{1}, \psi_{2}\right)$ is a subsolution of problem (5) if $\left(\psi_{1}, \psi_{2}\right) \in C^{2}(0,1) \times$ $C^{2}(0,1)$ satisfies

$$
\left\{\begin{array}{l}
\psi_{1}^{\prime \prime}(t)+a_{1}(t) f_{1}\left(\psi_{1}(t)+p_{\lambda, \mu}(t), \psi_{2}(t)+q_{\lambda, \mu}(t)\right) \geq 0, \quad t \in(0,1), \\
\psi_{2}^{\prime \prime}(t)+a_{2}(t) f_{2}\left(\psi_{1}(t)+p_{\lambda, \mu}(t), \psi_{2}(t)+q_{\lambda, \mu}(t)\right) \geq 0, \quad t \in(0,1), \\
\psi_{1}(0) \leq \delta, \quad \psi_{2}(0) \leq \delta \\
\psi_{1}(1)-\int_{0}^{1} g_{1}(s) \psi_{2}(s) d s \leq \delta \\
\psi_{2}(1)-\int_{0}^{1} g_{2}(s) \psi_{1}(s) d s \leq \delta .
\end{array}\right.
$$


We also say that $\left(\zeta_{1}, \zeta_{2}\right)$ is a supersolution of problem (5) if $\left(\zeta_{1}, \zeta_{2}\right) \in C^{2}(0,1) \times C^{2}(0,1)$ satisfies the reverse inequalities.

Theorem 2.1 Assume that there exist a subsolution $\left(\psi_{1}, \psi_{2}\right)$ and a supersolution $\left(\zeta_{1}, \zeta_{2}\right)$ of problem (5) such that $\left(\psi_{1}(t), \psi_{2}(t)\right) \leq\left(\zeta_{1}(t), \zeta_{2}(t)\right)$ for all $t \in[0,1]$. Then (5) has at least one solution $(u, v)$ such that

$$
\left(\psi_{1}(t), \psi_{2}(t)\right) \leq(u(t), v(t)) \leq\left(\zeta_{1}(t), \zeta_{2}(t)\right) \quad \text { for all } t \in[0,1] .
$$

Proof See Appendix.

To show the existence of a second positive solution of (4), we need the following lemmas for the fixed point index argument in [7].

Lemma 2.1 Let $X$ be a Banach space, let $P$ be cone in $X$, and let $\Omega$ be a bounded open set in $X$. Let $0 \in \Omega$, and let $T: P \cap \bar{\Omega} \rightarrow P$ be condensing. Suppose that $T x \neq \nu x$ for all $x \in P \cap \partial \Omega$ and all $v \geq 1$. Then

$$
i(T, P \cap \Omega, P)=1 .
$$

Lemma 2.2 Let $X$ be a Banach space, and let $P$ be a cone in $X$. For $r>0$, define $P_{r}=\{x \in$ $P:\|x\|<r\}$. Assume that $T: \bar{P}_{r} \rightarrow P$ is a compact map such that $T x \neq x$ for all $x \in \partial P_{r}$. If $\|x\| \leq\|T x\|$ for all $x \in \partial P_{r}$, then

$$
i\left(T, P_{r}, P\right)=0 .
$$

\section{Existence of multiple positive solutions}

We recall that $J=\left[j_{*}, j^{*}\right] \subset(0,1)$ is a closed interval of positive measure such that $a_{i}(t)>0$ for all $t \in J$ and $i=1,2$. Let $\gamma=\min \left\{j_{*}, 1-j^{*}\right\}>0$. Define the cone

$$
\mathcal{K}=\left\{\left(w_{1}, w_{2}\right) \in \mathcal{P}: \min _{J} w_{i}(t) \geq \gamma\left\|w_{i}\right\|_{\infty} \text { for } i=1,2\right\} .
$$

Then we have the following:

Lemma 3.1 For a given cone $\mathcal{P}$ in $X$, we have

$$
T_{\lambda, \mu, \delta}(\mathcal{P}) \subset \mathcal{K}
$$

Proof For any $\left(w_{1}, w_{2}\right) \in \mathcal{P}$, we first find

$$
\begin{aligned}
A_{\lambda, \mu, \delta}\left(w_{1}, w_{2}\right)(t) \leq & \delta\left(1+\rho v_{1}+\rho_{1} v_{2}\right) \\
& +\int_{0}^{1} G(s, s) a_{1}(s) f_{1}\left(w_{1}+p_{\lambda, \mu}, w_{2}+q_{\lambda, \mu}\right) d s \\
& +\rho_{1} \int_{0}^{1}\left(\int_{0}^{1} G(\tau, s) g_{2}(\tau) d \tau\right) a_{1}(s) f_{1}\left(w_{1}+p_{\lambda, \mu}, w_{2}+q_{\lambda, \mu}\right) d s \\
& +\rho \int_{0}^{1}\left(\int_{0}^{1} G(\tau, s) g_{1}(\tau) d \tau\right) a_{2}(s) f_{2}\left(w_{1}+p_{\lambda, \mu}, w_{2}+q_{\lambda, \mu}\right) d s
\end{aligned}
$$


for all $t \in[0,1]$. Thus we obtain

$$
\begin{aligned}
\left\|A_{\lambda, \mu, \delta}\left(w_{1}, w_{2}\right)\right\|_{\infty} \leq & \delta\left(1+\rho v_{1}+\rho_{1} v_{2}\right) \\
& +\int_{0}^{1} G(s, s) a_{1}(s) f_{1}\left(w_{1}+p_{\lambda, \mu}, w_{2}+q_{\lambda, \mu}\right) d s+\rho_{1} M_{1}+\rho M_{2},
\end{aligned}
$$

where

$$
M_{1}:=\int_{0}^{1}\left(\int_{0}^{1} G(\tau, s) g_{2}(\tau) d \tau\right) a_{1}(s) f_{1}\left(w_{1}+p_{\lambda, \mu}, w_{2}+q_{\lambda, \mu}\right) d s
$$

and

$$
M_{2}:=\int_{0}^{1}\left(\int_{0}^{1} G(\tau, s) g_{1}(\tau) d \tau\right) a_{2}(s) f_{2}\left(w_{1}+p_{\lambda, \mu}, w_{2}+q_{\lambda, \mu}\right) d s
$$

Similarly, we have

$$
\begin{aligned}
& \left\|B_{\lambda, \mu, \delta}\left(w_{1}, w_{2}\right)\right\|_{\infty} \\
& \quad \leq \delta\left(1+\rho v_{2}+\rho_{2} v_{1}\right) \\
& \quad+\int_{0}^{1} G(s, s) a_{2}(s) f_{2}\left(w_{1}+p_{\lambda, \mu}, w_{2}+q_{\lambda, \mu}\right) d s+\rho_{2} M_{2}+\rho M_{1} .
\end{aligned}
$$

Then by (9) we find that, for all $t \in J$,

$$
\begin{aligned}
A_{\lambda, \mu, \delta}\left(w_{1}, w_{2}\right)(t)= & \delta\left[1+\left(\rho v_{1}+\rho_{1} v_{2}\right) t\right] \\
& +\int_{0}^{1} G(t, s) a_{1}(s) f_{1}\left(w_{1}+p_{\lambda, \mu}, w_{2}+q_{\lambda, \mu}\right) d s+t \rho_{1} M_{1}+t \rho M_{2} \\
= & \delta\left[1+\left(\rho v_{1}+\rho_{1} v_{2}\right) t\right]+\int_{0}^{t} s(1-t) a_{1}(s) f_{1}\left(w_{1}+p_{\lambda, \mu}, w_{2}+q_{\lambda, \mu}\right) d s \\
& +\int_{t}^{1} t(1-s) a_{1}(s) f_{1}\left(w_{1}+p_{\lambda, \mu}, w_{2}+q_{\lambda, \mu}\right) d s+t \rho_{1} M_{1}+t \rho M_{2} \\
\geq & \delta\left[1+\left(\rho v_{1}+\rho_{1} v_{2}\right) j_{*}\right]+\left(1-j^{*}\right) \int_{0}^{t} s a_{1}(s) f_{1}\left(w_{1}+p_{\lambda, \mu}, w_{2}+q_{\lambda, \mu}\right) d s \\
& +j_{*} \int_{t}^{1}(1-s) a_{1}(s) f_{1}\left(w_{1}+p_{\lambda, \mu}, w_{2}+q_{\lambda, \mu}\right) d s+j_{*}\left(\rho_{1} M_{1}+\rho M_{2}\right) \\
\geq & \min \left\{j_{*}, 1-j^{*}\right\}\left(\delta\left(1+\rho v_{1}+\rho_{1} v_{2}\right)\right. \\
& +\int_{0}^{t} s(1-s) a_{1}(s) f_{1}\left(w_{1}+p_{\lambda, \mu}, w_{2}+q_{\lambda, \mu}\right) d s \\
& \left.+\int_{t}^{1} s(1-s) a_{1}(s) f_{1}\left(w_{1}+p_{\lambda, \mu}, w_{2}+q_{\lambda, \mu}\right) d s+\rho_{1} M_{1}+\rho M_{2}\right) \\
= & \min \left\{j_{*}, 1-j^{*}\right\}\left(\delta\left(1+\rho v_{1}+\rho_{1} v_{2}\right)\right.
\end{aligned}
$$




$$
\begin{aligned}
& \left.\quad+\int_{0}^{1} G(s, s) a_{1}(s) f_{1}\left(w_{1}+p_{\lambda, \mu}, w_{2}+q_{\lambda, \mu}\right) d s+\rho_{1} M_{1}+\rho M_{2}\right) \\
& \geq \gamma\left\|A_{\lambda, \mu}\left(w_{1}, w_{2}\right)\right\|_{\infty} .
\end{aligned}
$$

By the same argument, using (10), we also have that $B_{\lambda, \mu, \delta}\left(w_{1}, w_{2}\right)(t) \geq \gamma\left\|B_{\lambda, \mu}\left(w_{1}, w_{2}\right)\right\|_{\infty}$ for all $t \in J$.

As a consequence of the lemma, note that if $\left(w_{1}, w_{2}\right)$ is a solution of (5), then by (7) we have

$$
\inf _{t \in J} w_{i}(t) \geq \gamma\left\|w_{i}\right\|_{\infty} \quad \text { for } i=1,2
$$

Lemma 3.2 Assume $(\mathrm{H})$. If $(5)$ has a positive solution at $(\bar{\lambda}, \bar{\mu})$, then (5) also has a positive solution at $(\lambda, \mu)$ for all $(\lambda, \mu) \leq(\bar{\lambda}, \bar{\mu})$.

Proof Let $\left(\bar{w}_{1}, \bar{w}_{2}\right)$ be a positive solution of $(5)$ at $(\bar{\lambda}, \bar{\mu})$, and let $(\lambda, \mu) \in[0, \infty)^{2} \backslash\{(0,0)\}$ with $(\lambda, \mu) \leq(\bar{\lambda}, \bar{\mu})$. Then $\left(\bar{w}_{1}, \bar{w}_{2}\right)$ is a supersolution of (5) at $(\lambda, \mu)$ since $p_{\lambda, \mu}(t) \leq p_{\bar{\lambda}, \bar{\mu}}(t)$ and $q_{\lambda, \mu}(t) \leq q_{\bar{\lambda}, \bar{\mu}}(t)$ for $t \in(0,1)$ and $f_{i}$ is increasing for each $i=1,2$. Clearly, $(0,0)$ is a subsolution of (5). Notice that $(0,0)$ is not a solution of (5) since $f_{i}\left(p_{\lambda, \mu}(t), q_{\lambda, \mu}(t)\right)>0$ for $t \in(0,1)$ and $i=1,2$. Since $\left(\bar{w}_{1}, \bar{w}_{2}\right) \neq(0,0)$ and $\left(\bar{w}_{1}, \bar{w}_{2}\right) \geq(0,0)$, by Theorem 2.1 , (5) has a positive solution at $(\lambda, \mu)$.

Lemma 3.3 Assume $(\mathrm{H})$ and $(\mathrm{H} 1)$. Then there exists $(\tilde{\lambda}, \tilde{\mu})>(0,0)$ such that $(5)$ has a positive solution for all $(\lambda, \mu) \leq(\tilde{\lambda}, \tilde{\mu})$.

Proof Let $\left(\psi_{1}, \psi_{2}\right)$ be the unique solution of the following system:

$$
\begin{cases}\psi_{1}^{\prime \prime}(t)+a_{1}(t)=0 & t \in(0,1) \\ \psi_{2}^{\prime \prime}(t)+a_{2}(t)=0 & t \in(0,1) \\ \psi_{1}(0)=0=\psi_{2}(0), & \\ \psi_{1}(1)-\int_{0}^{1} g_{1}(s) \psi_{2}(s) d s=0, & \\ \psi_{2}(1)-\int_{0}^{1} g_{2}(s) \psi_{1}(s) d s=0 . & \end{cases}
$$

We recall that $p_{\lambda, \mu}(t)=\left(\rho \lambda+\rho_{1} \mu\right) t$ and $q_{\lambda, \mu}(t)=\left(\rho_{2} \lambda+\rho \mu\right) t$ and denote $\phi_{1}(t)=1+\left(\rho v_{1}+\right.$ $\left.\rho_{1} v_{2}\right) t$ and $\phi_{2}(t)=1+\left(\rho v_{2}+\rho_{2} v_{1}\right) t$. Then let $\alpha=\left\|\psi_{1}\right\|_{\infty}+\left\|\psi_{2}\right\|_{\infty}+\rho+\rho_{1}+\rho_{2}+\rho v_{2}+\rho_{2} v_{1}+$ $\rho v_{1}+\rho_{1} v_{2}+2$. By $(\mathrm{H} 1)$ there exist $\tilde{\lambda} \approx 0, \tilde{\mu} \approx 0$, and $\tilde{\delta} \approx 0$, sufficiently small, such that

$$
\begin{aligned}
f_{1}\left((\tilde{\lambda}+\tilde{\mu}+\tilde{\delta})\left\|\psi_{1}\right\|_{\infty}+p_{\tilde{\lambda}, \tilde{\mu}}(1)+\tilde{\delta} \phi_{1}(1),(\tilde{\lambda}+\tilde{\mu}+\tilde{\delta})\left\|\psi_{2}\right\|_{\infty}+q_{\tilde{\lambda}, \tilde{\mu}}(1)+\tilde{\delta} \phi_{2}(1)\right) \\
\leq \frac{1}{\alpha}\left((\tilde{\lambda}+\tilde{\mu}+\tilde{\delta})\left\|\psi_{1}\right\|_{\infty}+p_{\tilde{\lambda}, \tilde{\mu}}(1)+\tilde{\delta} \phi_{1}(1)\right. \\
\left.\quad+(\tilde{\lambda}+\tilde{\mu}+\tilde{\delta})\left\|\psi_{2}\right\|_{\infty}+q_{\tilde{\lambda}, \tilde{\mu}}(1)+\tilde{\delta} \phi_{2}(1)\right) .
\end{aligned}
$$

Now we define $Z_{1}(t)=(\tilde{\lambda}+\tilde{\mu}+\tilde{\delta}) \psi_{1}+\tilde{\delta} \phi_{1}(t)$ and $Z_{2}(t)=(\tilde{\lambda}+\tilde{\mu}+\tilde{\delta}) \psi_{2}+\tilde{\delta} \phi_{2}(t)$. Then, from (H) and (12) we have $Z_{1}(0)=\tilde{\delta}$,

$$
\begin{aligned}
& Z_{1}^{\prime \prime}(t)+a_{1}(t) f_{1}\left(Z_{1}(t)+p_{\tilde{\lambda}, \tilde{\mu}}(t), Z_{2}(t)+q_{\tilde{\lambda}, \tilde{\mu}}(t)\right) \\
& \quad=-(\tilde{\lambda}+\tilde{\mu}+\tilde{\delta}) a_{1}(t)+a_{1}(t) f_{1}\left(Z_{1}(t)+p_{\tilde{\lambda}, \tilde{\mu}}(t), Z_{2}(t)+q_{\tilde{\lambda}, \tilde{\mu}}(t)\right)
\end{aligned}
$$




$$
\begin{aligned}
\leq & a_{1}(t)\left\{f _ { 1 } \left((\tilde{\lambda}+\tilde{\mu}+\tilde{\delta})\left\|\psi_{1}\right\|_{\infty}+p_{\tilde{\lambda}, \tilde{\mu}}(1)+\tilde{\delta} \phi_{1}(1),\right.\right. \\
& \left.\left.(\tilde{\lambda}+\tilde{\mu}+\tilde{\delta})\left\|\psi_{2}\right\|_{\infty}+q_{\tilde{\lambda}, \tilde{\mu}}(1)+\tilde{\delta} \phi_{2}(1)\right)-(\tilde{\lambda}+\tilde{\mu}+\tilde{\delta})\right\} \\
\leq & a_{1}(t)\left[\frac { 1 } { \alpha } \left((\tilde{\lambda}+\tilde{\mu}+\tilde{\delta})\left\|\psi_{1}\right\|_{\infty}+p_{\tilde{\lambda}, \tilde{\mu}}(1)+\tilde{\delta} \phi_{1}(1)\right.\right. \\
& \left.\left.+(\tilde{\lambda}+\tilde{\mu}+\tilde{\delta})\left\|\psi_{2}\right\|_{\infty}+q_{\tilde{\lambda}, \tilde{\mu}}(1)+\tilde{\delta} \phi_{2}(1)\right)-(\tilde{\lambda}+\tilde{\mu}+\tilde{\delta})\right]
\end{aligned}
$$

$\leq 0$

and

$$
Z_{1}(1)-\int_{0}^{1} g_{1}(s) Z_{2}(s) d s=\tilde{\delta} \phi_{1}(1)-\tilde{\delta} \int_{0}^{1} g_{1}(s) \phi_{2}(s) d s=\tilde{\delta} \geq \delta .
$$

Similarly, we can show that $Z_{2}$ satisfies the same inequalities. This shows that $\left(Z_{1}, Z_{2}\right)$ is a supersolution of $(5)$ at $(\tilde{\lambda}, \tilde{\mu})$. On the other hand, $(0,0)$ is a strict subsolution such that $(0,0) \leq\left(Z_{1}, Z_{2}\right)$ in $[0,1]$. Hence, by Theorem $2.1,(5)$ has a positive solution at $(\tilde{\lambda}, \tilde{\mu})$, and then Lemma 3.2 completes the proof.

Lemma 3.4 Assume (H2). There exists $M>0$ such that $\left\|\left(w_{1}, w_{2}\right)\right\| \leq M$ for all possible solutions $\left(w_{1}, w_{2}\right)$ of $(5)$.

Proof As $\left(w_{1}, w_{2}\right)$ is a solution of (5), we find

$$
\begin{aligned}
\left\|w_{1}\right\|_{\infty} \geq & w_{1}\left(j_{*}\right) \\
= & \delta\left[1+\left(\rho v_{1}+\rho_{1} v_{2}\right) j_{*}\right] \\
& +\int_{0}^{1}\left(G\left(j_{*}, s\right)+\rho_{1} j_{*} \int_{0}^{1} G(\tau, s) g_{2}(\tau) d \tau\right) a_{1}(s) f_{1}\left(w_{1}+p_{\lambda, \mu}, w_{2}+q_{\lambda, \mu}\right) \\
& +\left(\rho j_{*} \int_{0}^{1} G(\tau, s) g_{1}(\tau) d \tau\right) a_{2}(s) f_{2}\left(w_{1}+p_{\lambda, \mu}, w_{2}+q_{\lambda, \mu}\right) d s \\
\geq & \int_{j_{*}}^{j^{*}}\left(\rho j_{*} \int_{j_{*}}^{j^{*}} G(\tau, s) g_{1}(\tau) d \tau\right) a_{2}(s) f_{2}\left(w_{1}+p_{\lambda, \mu}, w_{2}+q_{\lambda, \mu}\right) d s \\
\geq & \rho j_{*} \int_{j_{*}}^{j^{*}} g_{1}(s)\left(\int_{j_{*}}^{s} \tau(1-s) a_{2}(\tau) f_{2}\left(w_{1}+p_{\lambda, \mu}, w_{2}+q_{\lambda, \mu}\right) d \tau\right. \\
& \left.+\int_{s}^{j^{*}} s(1-\tau) a_{2}(\tau) f_{2}\left(w_{1}+p_{\lambda, \mu}, w_{2}+q_{\lambda, \mu}\right) d \tau\right) d s \\
\geq & \rho j_{*} \int_{j_{*}}^{j^{*}} g_{1}(s) j_{*}\left(1-j^{*}\right)\left(\int_{j_{*}}^{j^{*}} a_{2}(\tau) f_{2}\left(w_{1}+p_{\lambda, \mu}, w_{2}+q_{\lambda, \mu}\right) d \tau\right) d s \\
\geq & \rho j_{*}^{2}\left(1-j^{*}\right)\left(\int_{j_{*}}^{j^{*}} g_{1}(s) d s\right)\left(\int_{j_{*}}^{j^{*}} a_{2}(s) d s\right) f_{2}\left(\gamma\left\|w_{1}\right\|_{\infty}, \gamma\left\|w_{2}\right\|_{\infty}\right) .
\end{aligned}
$$

Hence we obtain

$$
\left\|w_{1}\right\|_{\infty}+\left\|w_{2}\right\|_{\infty} \geq \gamma^{2} \rho j_{*}\left(\int_{j_{*}}^{j^{*}} g_{1}(s) d s\right)\left(\int_{j_{*}}^{j^{*}} a_{2}(s) d s\right) f_{2}\left(\gamma\left\|w_{1}\right\|_{\infty}, \gamma\left\|w_{2}\right\|_{\infty}\right),
$$


which implies that

$$
\left(\gamma^{3} \rho j_{*}\right)^{-1}\left(\int_{j_{*}}^{j_{*}^{*}} g_{1}(s) d s\right)^{-1}\left(\int_{j_{*}}^{j^{*}} a_{2}(s) d s\right)^{-1} \geq \frac{f_{2}\left(\gamma\left\|w_{1}\right\|_{\infty}, \gamma\left\|w_{2}\right\|_{\infty}\right)}{\gamma\left\|w_{1}\right\|_{\infty}+\gamma\left\|w_{2}\right\|_{\infty}}
$$

If $\left\|w_{1}\right\|_{\infty} \rightarrow \infty$, then (13) contradict to (H2). Similarly, we can show that $\left\|w_{2}\right\|_{\infty}$ is bounded.

Define $\mathcal{S}=\left\{(\lambda, \mu) \in[0, \infty)^{2} \backslash\{(0,0)\}:(5)\right.$ has a positive solution at $\left.(\lambda, \mu)\right\}$. Then $\mathcal{S} \neq \emptyset$ by Lemma 3.3 .

Lemma 3.5 Assume $(\mathrm{H})$ and $(\mathrm{H} 2)$. Then $(\mathcal{S}, \leq)$ is bounded above.

Proof We claim that there exist $\bar{\lambda}, \bar{\mu}>0$ such that (5) has no solution for $\lambda>\bar{\lambda}$ or $\mu>\bar{\mu}$. Suppose on the contrary that there exists a sequence $\left(\lambda_{n}, \mu_{n}\right)$ such that either $\lambda_{n} \rightarrow \infty$ or $\mu_{n} \rightarrow \infty$ and (5) has a positive solution $\left(w_{1 n}, w_{2 n}\right)$ at $\lambda=\lambda_{n}$ and $\mu=\mu_{n}$. Without loss of generality, we assume that $\lambda_{n} \rightarrow \infty$. First, we observe from (H2) that there exists $R_{f_{2}}$ sufficiently large such that

$$
f_{2}(u, v) \geq u+v \quad \text { for all } u+v \geq R_{f_{2}} .
$$

Now we choose $\lambda_{n}$ sufficiently large such that

$$
\gamma\left(\left\|w_{1 n}\right\|_{\infty}+\left\|w_{2 n}\right\|_{\infty}\right)+j_{*}\left(\rho+\rho_{2}\right) \lambda_{n}+j_{*}\left(\rho_{1}+\rho\right) \mu_{n} \geq R_{f_{2}} .
$$

Then, for such $\lambda_{n}$, from (14) it follows that

$$
\begin{gathered}
f_{2}\left(\gamma\left\|w_{1 n}\right\|_{\infty}+\left(\rho \lambda_{n}+\rho_{1} \mu_{n}\right) j_{*}, \gamma\left\|w_{2 n}\right\|_{\infty}+\left(\rho_{2} \lambda_{n}+\rho \mu_{n}\right) j_{*}\right) \\
\geq \gamma\left(\left\|w_{1 n}\right\|_{\infty}+\left\|w_{2 n}\right\|_{\infty}\right)+j_{*}\left(\rho+\rho_{2}\right) \lambda_{n}+j_{*}\left(\rho_{1}+\rho\right) \mu_{n} .
\end{gathered}
$$

Since $w_{1 n}$ is a solution of (5), using (3), (11), and (15), we have

$$
\begin{aligned}
\left\|w_{1 n}\right\|_{\infty} \geq & w_{1 n}\left(j_{*}\right) \\
= & \delta\left[1+\left(\rho v_{1}+\rho_{1} v_{2}\right) j_{*}\right] \\
& +\int_{0}^{1} H_{2}\left(j_{*}, s\right) a_{1}(s) f_{1}\left(w_{1 n}+p_{\lambda_{n}, \mu_{n}}, w_{2 n}+q_{\lambda_{n}, \mu_{n}}\right) \\
& +K_{1}\left(j_{*}, s\right) a_{2}(s) f_{2}\left(w_{1 n}+p_{\lambda_{n}, \mu_{n}}, w_{2 n}+q_{\lambda_{n}, \mu_{n}}\right) d s \\
\geq & \int_{j_{*}}^{j^{*}} K_{1}\left(j_{*}, s\right) a_{2}(s) f_{2}\left(\gamma\left\|w_{1 n}\right\|_{\infty}+p_{\lambda_{n}, \mu_{n}}\left(j_{*}\right), \gamma\left\|w_{2 n}\right\|_{\infty}+q_{\lambda_{n}, \mu_{n}}\left(j_{*}\right)\right) d s \\
\geq & \left(\gamma\left(\left\|w_{1 n}\right\|_{\infty}+\left\|w_{2 n}\right\|_{\infty}\right)+j_{*}\left(\rho+\rho_{2}\right) \lambda_{n}\right. \\
& \left.+j_{*}\left(\rho_{1}+\rho\right) \mu_{n}\right) \int_{j_{*}}^{j^{*}}\left(\rho j_{*} \int_{0}^{1} G(\tau, s) g_{1}(\tau) d \tau\right) a_{2}(s) d s,
\end{aligned}
$$

which implies that $\left\|w_{1 n}\right\|_{\infty} \rightarrow \infty$, a contradiction to Lemma 3.4. Hence, we conclude that there exist $\bar{\lambda}, \bar{\mu}>0$ such that (5) has no solution for $\lambda>\bar{\lambda}$ or $\mu>\bar{\mu}$. 
The following Lemmas 3.6, 3.7, and 3.8 can be proved by the same ideas as in Lemma 3.5, Lemma 3.6, and Theorem 3.1 in [9], respectively.

Lemma 3.6 Assume $(\mathrm{H})$ and $(\mathrm{H} 2)$. Then every chain in $\mathcal{S}$ has a unique supremum in $\mathcal{S}$.

Lemma 3.7 Assume $(\mathrm{H})$ and $(\mathrm{H} 2)$. Then there exists $\mu^{*} \in[\tilde{\mu}, \bar{\mu}]$ such that (5) has a positive solution at $(0, \mu)$ for all $0<\mu \leq \mu^{*}$ and no solution at $(0, \mu)$ for all $\mu>\mu^{*}$. Similarly, there exists $\lambda^{*} \in[\tilde{\lambda}, \bar{\lambda}]$ such that (5) has a positive solution at $(\lambda, 0)$ for all $0<\lambda \leq \lambda^{*}$ and no solution at $(\lambda, 0)$ for all $\lambda>\lambda^{*}$.

Lemma 3.8 Assume $(\mathrm{H} 1)$ and $(\mathrm{H} 2)$. Then there exists a continuous curve $\Gamma_{\delta}$ splitting $[0, \infty)^{2} \backslash\{(0,0)\}$ into two disjoint subsets $\Theta_{\delta, 1}$ and $\Theta_{\delta, 2}$ such that $(5)$ has at least one positive solution if $(\lambda, \mu) \in \Theta_{1} \cup \Gamma_{\delta}$ and no positive solution if $(\lambda, \mu) \in \Theta_{\delta, 2}$.

Notice that by the sub- and supersolution theorem we can show that if $0<\bar{\delta}<\delta$, then $\Theta_{\delta, 1} \cup \Gamma_{\delta} \subset \Theta_{\bar{\delta}, 1} \cup \Gamma_{\bar{\delta}}$. Let $\Theta=\bigcup_{\delta>0}\left(\Theta_{\delta, 1} \cup \Gamma_{\delta}\right)$. Then $\Theta \subset \Theta_{0,1}$. Now we prove our main theorem, Theorem 1.1, by taking $\Theta_{1}=\Theta_{0,1}, \Theta_{2}=\Theta_{0,2}$, and $\Gamma=\Gamma_{0}$ in Theorem 3.1.

Theorem 3.1 Assume $(\mathrm{H}),(\mathrm{H} 1)$, and $(\mathrm{H} 2)$. Then there exists a continuous curve $\Gamma_{0}$ splitting $[0, \infty)^{2} \backslash\{(0,0)\}$ into two disjoint subsets $\Theta_{0,1}, \Theta_{0,2}$ and a subset $\Theta \subset \Theta_{0,1}$ such that (4) has at least two positive solutions for $(\lambda, \mu) \in \Theta$, at least one positive solution for $(\lambda, \mu) \in\left(\Theta_{0,1} \backslash \Theta\right) \cup \Gamma_{0}$, and no positive solution for $(\lambda, \mu) \in \Theta_{0,2}$.

Proof We prove that (4) has a positive second solution for $(\lambda, \mu) \in \Theta$. If $(\lambda, \mu) \in \Theta$, then there exists $\delta>0$ such that $(\lambda, \mu) \in\left(\Theta_{\delta, 1} \cup \Gamma_{\delta}\right)$. Now we let $\left(w_{\delta, 1}, w_{\delta, 2}\right)$ be a positive solution of (5) at $(\lambda, \mu)$ and define $\Omega=\left\{\left(w_{1}, w_{2}\right) \in X:-\epsilon<w_{1}(t)<w_{\delta, 1}(t),-\epsilon<w_{2}(t)<w_{\delta, 2}(t), t \in\right.$ $[0,1]\}$. Then $\Omega$ is a bounded open set in $X$ such that $0 \in \Omega$. Here we denote

$$
\left(A_{\lambda, \mu}\left(w_{1}, w_{2}\right)(t), B_{\lambda, \mu}\left(w_{1}, w_{2}\right)(t)\right):=\left(A_{\lambda, \mu, 0}\left(w_{1}, w_{2}\right)(t), B_{\lambda, \mu, 0}\left(w_{1}, w_{2}\right)(t)\right)
$$

and

$$
T_{\lambda, \mu}\left(w_{1}, w_{2}\right)(t):=T_{\lambda, \mu, 0}\left(w_{1}, w_{2}\right)(t)
$$

Then $T_{\lambda, \mu}: \mathcal{K} \cap \bar{\Omega} \rightarrow \mathcal{K}$ is condensing. Let $\left(w_{1}, w_{2}\right) \in \mathcal{K} \cap \partial \Omega$. Then there exists $t_{0} \in[0,1]$ such that either $w_{1}\left(t_{0}\right)=w_{\delta, 1}\left(t_{0}\right)$ or $w_{2}\left(t_{0}\right)=w_{\delta, 2}\left(t_{0}\right)$. Suppose that $w_{1}\left(t_{0}\right)=w_{\delta, 1}\left(t_{0}\right)$. Then by $(\mathrm{H})$

$$
\begin{aligned}
A_{\lambda, \mu}\left(w_{1}, w_{2}\right)\left(t_{0}\right)= & \int_{0}^{1} H_{2}\left(t_{0}, s\right) a_{1}(s) f_{1}\left(w_{1}+p_{\lambda, \mu}, w_{2}+q_{\lambda, \mu}\right) \\
& +K_{1}\left(t_{0}, s\right) a_{2}(s) f_{2}\left(w_{1}+p_{\lambda, \mu}, w_{2}+q_{\lambda, \mu}\right) d s \\
< & \delta\left[1+\left(\rho v_{1}+\rho_{1} v_{2}\right) t_{0}\right] \\
& +\int_{0}^{1} H_{2}\left(t_{0}, s\right) a_{1}(s) f_{1}\left(w_{\delta, 1}+p_{\lambda, \mu}, w_{\delta, 2}+q_{\lambda, \mu}\right) \\
& +K_{1}\left(t_{0}, s\right) a_{2}(s) f_{2}\left(w_{\delta, 1}+p_{\lambda, \mu}, w_{\delta, 2}+q_{\lambda, \mu}\right) d s
\end{aligned}
$$




$$
\begin{aligned}
& =w_{\delta, 1}\left(t_{0}\right) \\
& =w_{1}\left(t_{0}\right) \leq v w_{1}\left(t_{0}\right)
\end{aligned}
$$

for all $v \geq 1$. Thus $T_{\lambda, \mu}\left(w_{1}, w_{2}\right) \neq v\left(w_{1}, w_{2}\right)$ for all $\left(w_{1}, w_{2}\right) \in \mathcal{K} \cap \partial \Omega$ and $v \geq 1$. By Lemma 2.1 we conclude

$$
i\left(T_{\lambda, \mu}, \mathcal{K} \cap \Omega, \mathcal{K}\right)=1
$$

Next, we denote $\kappa=\min _{J \times J} K_{1}(t, s)$. By $(\mathrm{H} 2)$ there exists $R_{f_{2}}>0$ sufficiently large such that

$$
f_{2}(u, v) \geq \eta(u+v) \quad \text { for } u+v \geq R_{f_{2}}
$$

where $\eta>0$ can be chosen such that $\eta \kappa \gamma \int_{j_{*}}^{j^{*}} a_{2}(s) d s>1$. Let $R=\max \left\{M+1, \frac{1}{\gamma} R_{f_{2}}\right\}$, where $M$ is defined in Lemma 3.4. Let $\mathcal{K}_{R}=\left\{\left(w_{1}, w_{2}\right) \in \mathcal{K}:\left\|\left(w_{1}, w_{2}\right)\right\|<R\right\}$. Then, by Lemma 3.4, $\left(w_{1}, w_{2}\right) \neq T_{\lambda, \mu}\left(w_{1}, w_{2}\right)$ for $\left(w_{1}, w_{2}\right) \in \partial \mathcal{K}_{R}$. Moreover, if $\left(w_{1}, w_{2}\right) \in \partial \mathcal{K}_{R}$, then

$$
\min _{J}\left(w_{1}(t)+w_{2}(t)\right) \geq \gamma\left(\left\|w_{1}\right\|_{\infty}+\left\|w_{2}\right\|_{\infty}\right) \geq R_{f_{2}}
$$

which implies that $f_{2}\left(w_{1}(t), w_{2}(t)\right) \geq \eta\left(w_{1}(t)+w_{2}(t)\right)$ for all $t \in J$. Finally, we find that, for $\left(w_{1}, w_{2}\right) \in \partial \mathcal{K}_{R}$,

$$
\begin{aligned}
A_{\lambda, \mu}\left(w_{1}, w_{2}\right)\left(j_{*}\right)= & \int_{0}^{1} H_{2}\left(j_{*}, s\right) a_{1}(s) f_{1}\left(w_{1}+p_{\lambda, \mu}, w_{2}, q_{\lambda, \mu}\right) \\
& +K_{1}\left(j_{*}, s\right) a_{2}(s) f_{2}\left(w_{1}+p_{\lambda, \mu}, w_{2}+q_{\lambda, \mu}\right) d s \\
\geq & \kappa \int_{j_{*}}^{j^{*}} a_{2}(s) f_{2}\left(w_{1}+p_{\lambda, \mu}, w_{2}+q_{\lambda, \mu}\right) d s \\
\geq & \kappa \eta \int_{j_{*}}^{j^{*}} a_{2}(s)\left(w_{1}(s)+w_{2}(s)\right) d s \\
\geq & \kappa \eta \gamma\left\|\left(w_{1}, w_{2}\right)\right\| \int_{j_{*}}^{j^{*}} a_{2}(s) d s \\
> & \left\|\left(w_{1}, w_{2}\right)\right\| .
\end{aligned}
$$

Therefore $\left\|T_{\lambda, \mu}\left(w_{1}, w_{2}\right)\right\| \geq\left\|A_{\lambda, \mu}\left(w_{1}, w_{2}\right)\right\|_{\infty} \geq A_{\lambda, \mu}\left(w_{1}, w_{2}\right)\left(j_{*}\right)>\left\|\left(w_{1}, w_{2}\right)\right\|$, and by Lemma 2.2 we find

$$
i\left(T_{\lambda, \mu}, \mathcal{K}_{R}, \mathcal{K}\right)=0
$$

By the additivity of the fixed point index we obtain

$$
0=i\left(T_{\lambda, \mu}, \mathcal{K}_{R}, \mathcal{K}\right)=i\left(T_{\lambda, \mu}, \mathcal{K} \cap \Omega, \mathcal{K}\right)+i\left(T_{\lambda, \mu}, \mathcal{K}_{R} \backslash \overline{\mathcal{K} \cap \Omega}, \mathcal{K}\right)
$$

Since $i\left(T_{\lambda, \mu}, \mathcal{K} \cap \Omega, \mathcal{K}\right)=1$, we find $i\left(T_{\lambda, \mu}, \mathcal{K}_{R} \backslash \overline{\mathcal{K} \cap \Omega}, \mathcal{K}\right)=-1$, which implies that $T_{\lambda, \mu}$ has a fixed point on $\mathcal{K} \cap \Omega$ and on $\mathcal{K}_{R} \backslash \overline{\mathcal{K} \cap \Omega}$. Hence the proof is completed.

Now, we give a simple example for the main results. 
Example 3.2 Consider $N=3$ and $r_{0}=1$ for system (1). Then $\Omega_{e}=\left\{x \in(-\infty, \infty)^{3}:|x| \geq\right.$ 1\}. Let $K_{i}(r)=r^{-\alpha_{i}}$ for $r \in(1, \infty)$, where $\alpha_{i}>2$ for $i=1,2$. Then there exists $v$ such that $1<\nu<\min \left\{\alpha_{1}, \alpha_{2}\right\}-1$, and hence we get that $\nu-\alpha_{i}<-1$ and $\int_{1}^{\infty} r^{\nu} K_{i}(r) d r=\int_{1}^{\infty} r^{\nu-\alpha_{i}} d r<$ $\infty$ for $i=1,2$. Let $l_{i}(r)=r^{-\beta_{i}}$ for $r \in(1, \infty)$, where $\beta_{i}>4 \pi+2(i=1,2)$. It is easy to see that $w_{3} \int_{1}^{\infty} r l_{i}(r) d r=4 \pi \int_{1}^{\infty} r^{1-\beta_{i}} d r=\frac{4 \pi}{\beta_{i}-2}<1$ as $w_{3}=4 \pi$. Now, if $f_{1}(u, v)=u^{2}+v^{3}$ and $f_{2}(u, v)=e^{u+v}-(u+v)$, then $(\mathrm{H}),(\mathrm{H} 1)$, and (H2) hold for $f_{1}$ and $f_{2}$. Thus the conclusion of Theorem 1.1 is valid. We note that, for the corresponding radial transformed problem (2) of (1), the conclusion of Theorem 3.1 is also valid.

\section{Appendix: sub- and supersolution theorem}

In this subsection, we prove Theorem 2.1. We consider the system with more general boundary conditions:

$$
\left\{\begin{array}{l}
u^{\prime \prime}(t)+a_{1}(t) f_{1}(u(t), v(t))=0, \quad t \in(0,1), \\
v^{\prime \prime}(t)+a_{2}(t) f_{2}(u(t), v(t))=0, \quad t \in(0,1), \\
u(0)=a, v(0)=b, \\
u(1)-\int_{0}^{1} g_{1}(s) v(s) d s=\lambda, \\
v(1)-\int_{0}^{1} g_{2}(s) u(s) d s=\mu,
\end{array}\right.
$$

where $\lambda, \mu, a$, and $b$ are constants.

Definition 4.1 We say that $\left(\psi_{1}, \psi_{2}\right) \in C^{2}(0,1) \times C^{2}(0,1)$ is a subsolution of problem (16) if

$$
\left\{\begin{array}{l}
\psi_{1}^{\prime \prime}(t)+a_{1}(t) f_{1}\left(\psi_{1}(t), \psi_{2}(t)\right) \geq 0, \quad t \in(0,1) \\
\psi_{2}^{\prime \prime}(t)+a_{2}(t) f_{2}\left(\psi_{1}(t), \psi_{2}(t)\right) \geq 0, \quad t \in(0,1) \\
\psi_{1}(0) \leq a, \psi_{2}(0) \leq b \\
\psi_{1}(1)-\int_{0}^{1} g_{1}(s) \psi_{2}(s) d s \leq \lambda \\
\psi_{2}(1)-\int_{0}^{1} g_{2}(s) \psi_{1}(s) d s \leq \mu
\end{array}\right.
$$

We also say that $\left(\zeta_{1}, \zeta_{2}\right) \in C^{2}(0,1) \times C^{2}(0,1)$ is an supersolution of problem (16) if the reverse inequalities are satisfied.

A theorem for sub- and supersolutions to problem (16) is as follows.

Theorem 4.1 Assume that $f_{1}(t, s)$ and $f_{2}(t, s)$ is quasimonotone increasing with respect to $s$ and $t$, respectively (i.e., $f_{1}\left(t, s_{1}\right) \leq f_{1}\left(t, s_{2}\right)$ for $s_{1} \leq s_{2}$ and $f_{2}\left(t_{1}, s\right) \leq f_{2}\left(t_{2}, s\right)$ for $\left.t_{1} \leq t_{2}\right)$ and there exist a subsolution $\left(\psi_{1}, \psi_{2}\right)$ and a supersolution $\left(\zeta_{1}, \zeta_{2}\right)$ of problem (16) such that $\left(\psi_{1}(t), \psi_{2}(t)\right) \leq\left(\zeta_{1}(t), \zeta_{2}(t)\right)$ for all $t \in[0,1]$. Then problem (16) has at least one solution $(u, v)$ such that

$$
\left(\psi_{1}(t), \psi_{2}(t)\right) \leq(u(t), v(t)) \leq\left(\zeta_{1}(t), \zeta_{2}(t)\right) \text { for all } t \in[0,1]
$$


Proof Define $\gamma_{i}:[0,1] \times(-\infty, \infty) \rightarrow[0, \infty)$ for $i=1,2$ by

$$
\gamma_{i}(t, s):= \begin{cases}\zeta_{i}(t), & s \geq \zeta_{i}(t) \\ s, & \psi_{i}(t) \leq s \leq \zeta_{i}(t) \\ \psi_{i}(t), & s \leq \psi_{i}(t)\end{cases}
$$

and consider the modified problem

$$
\left\{\begin{array}{l}
u^{\prime \prime}(t)+a_{1}(t) f_{1}\left(\gamma_{1}(t, u(t)), \gamma_{2}(t, v(t))\right)=0, \quad t \in(0,1), \\
v^{\prime \prime}(t)+a_{2}(t) f_{2}\left(\gamma_{1}(t, u(t)), \gamma_{2}(t, v(t))\right)=0, \quad t \in(0,1), \\
u(0)=a, \quad v(0)=b, \\
u(1)-\int_{0}^{1} g_{1}(s) \gamma_{2}(s, v(s)) d s=\lambda, \\
v(1)-\int_{0}^{1} g_{2}(s) \gamma_{1}(s, u(s)) d s=\mu .
\end{array}\right.
$$

For given $(w, z) \in C[0,1] \times C[0,1]$, define $g_{w, z}:(-\infty, \infty)^{2} \rightarrow(-\infty, \infty)^{2}$ by

$$
\begin{aligned}
g_{w, z}(x, y):= & \left(\int_{0}^{1} g_{1}(s) \gamma_{2}(s, z(s)+y s+b) d s+\lambda-a,\right. \\
& \left.\int_{0}^{1} g_{2}(s) \gamma_{1}(s, w(s)+x s+a) d s+\mu-b\right)
\end{aligned}
$$

for $(x, y) \in(-\infty, \infty)^{2}$. Since $\left|\gamma_{i}(s, x)-\gamma_{i}(s, y)\right| \leq|x-y|$ for any $x, y \in(-\infty, \infty)$ and $s \in[0,1]$, $g_{w, z}$ is a contraction mapping on $(-\infty, \infty)^{2}$, and thus there exists a unique fixed point $\left(A_{w, z}, B_{w, z}\right) \in(-\infty, \infty)^{2}$ of $g_{w, z}$, which satisfies

$$
\begin{aligned}
\left(A_{w, z}, B_{w, z}\right)= & \left(\int_{0}^{1} g_{1}(s) \gamma_{2}\left(s, z(s)+B_{w, z} s+b\right) d s+\lambda-a,\right. \\
& \left.\int_{0}^{1} g_{2}(s) \gamma_{1}\left(s, w(s)+A_{w, z} s+a\right) d s+\mu-b\right) .
\end{aligned}
$$

If $(w, z)$ is a solution of

$$
\left\{\begin{array}{l}
w^{\prime \prime}(t)+a_{1}(t) f_{1}\left(\gamma_{1}\left(t, w(t)+A_{w, z} t+a\right), \gamma_{2}\left(t, z(t)+B_{w, z} t+b\right)\right)=0 \\
z^{\prime \prime}(t)+a_{2}(t) f_{2}\left(\gamma_{1}\left(t, w(t)+A_{w, z} t+a\right), \gamma_{2}\left(t, z(t)+B_{w, z} t+b\right)\right)=0 \\
w(0)=w(1)=z(0)=z(1)=0
\end{array}\right.
$$

then $(u(t), v(t)):=\left(w(t)+A_{w, z} t+a, z(t)+B_{w, z} t+b\right)$ is a solution of (17).

Now, we define $T: \mathcal{P} \rightarrow \mathcal{P}$ by

$$
T(w, z)(t):=\left(T_{1}(w, z)(t), T_{2}(w, z)(t)\right)
$$

where

$$
T_{1}(w, z)(t)=\int_{0}^{1} G(t, s) a_{1}(s) f_{1}\left(\gamma_{1}\left(s, w(s)+A_{w, z} s+a\right), \gamma_{2}\left(s, z(s)+B_{w, z} s+b\right)\right) d s,
$$




$$
T_{2}(w, z)(t)=\int_{0}^{1} G(t, s) a_{2}(s) f_{2}\left(\gamma_{1}\left(s, w(s)+A_{w, z} s+a\right), \gamma_{2}\left(s, z(s)+B_{w, z} s+b\right)\right) d s .
$$

Then $(w, z)$ is a fixed point of $T$ in $\mathcal{P}$ if and only if $(w, z)$ is a nonnegative solution of (18). Since $T_{\gamma}$ is completely continuous on $\mathcal{P}$ and $T(\mathcal{P})$ is bounded in $C[0,1] \times C[0,1], T$ has a fixed point $(w, z)$, and consequently $(17)$ has a nonnegative solution $(u, v)$. Now if we prove that $\left(\psi_{1}(t), \psi_{2}(t)\right) \leq(u(t), v(t)) \leq\left(\zeta_{1}(t), \zeta_{2}(t)\right)$ for $t \in[0,1]$, then, by the definition of $\gamma_{i},(16)$ has a solution $(u, v)$ such that $\left(\psi_{1}(t), \psi_{2}(t)\right) \leq(u(t), v(t)) \leq\left(\zeta_{1}(t), \zeta_{2}(t)\right)$ for all $t \in[0,1]$, and the proof is done.

To show that $u(t) \leq \zeta_{1}(t)$, we set $X(t):=u(t)-\zeta_{1}(t)$. Note that

$$
\begin{aligned}
& X(0)=u(0)-\zeta_{1}(0) \leq 0, \\
& X(1)=u(1)-\zeta_{1}(1) \leq \int_{0}^{1} g_{1}(s)\left[\gamma_{2}(s, v(s))-\zeta_{2}(s)\right] d s \leq 0 .
\end{aligned}
$$

Now let us assume on the contrary that there is $t_{0} \in(0,1)$ such that $X\left(t_{0}\right)=u\left(t_{0}\right)-\zeta_{1}\left(t_{0}\right)>0$. It is clear that there exists $\sigma \in(0,1)$ such that $X(\sigma)=\max _{t \in[0,1]} X(t)>0$. Then $X^{\prime}(\sigma)=0$, and there is $a \in(\sigma, 1)$ such that $X^{\prime}(t)<0$ and $X(t)>0$ for $t \in(\sigma, a]$, which means that

$$
u^{\prime}(\sigma)=\zeta_{1}^{\prime}(\sigma), \quad u^{\prime}(t)<\zeta_{1}^{\prime}(t), \quad \text { and } \quad u(t)>\zeta_{1}(t) \quad \text { for } t \in(\sigma, a]
$$

By the quasimonotonicity of $f_{1}(t, s)$ with respect to $s$ and the definition of $\gamma_{i}$ for $i=1,2$, we obtain

$$
\begin{aligned}
-u^{\prime \prime}(t) & =a_{1}(t) f_{1}\left(\gamma_{1}(t, u(t)), \gamma_{2}(t, v(t))\right) \\
& \leq a_{1}(t) f_{1}\left(\zeta_{1}(t), \zeta_{2}(t)\right) \leq-\zeta_{1}^{\prime \prime}(t) \quad \text { for } t \in(\sigma, a] .
\end{aligned}
$$

Integrating this from $\sigma$ to $t \in(\sigma, a]$, we find

$$
u^{\prime}(t) \geq \zeta_{1}^{\prime}(t), \quad \text { for } t \in(\sigma, a]
$$

which contradicts to (19) Thus $u(t) \leq \zeta_{1}(t)$ for $t \in[0,1]$. In a similar manner, we can prove that $\psi_{1}(t) \leq u(t), v(t) \leq \zeta_{2}(t)$ and $\psi_{2}(t) \leq v(t)$ for $t \in[0,1]$, and hence the proof is complete.

Acknowledgements

Both authors thank the anonymous reviewers for carefully reading this paper and constructive comments.

\section{Funding}

E. Ko was supported by the National Research Foundation of Korea (NRF) grant funded by the Korea Government (NRF-2017R1D1A1B03030681). E. K. Lee was supported by a 2-year Research Grant of Pusan National University.

\section{Availability of data and materials}

Not applicable.

\section{Competing interests}

The authors declare that they have no competing interests 


\section{Author details}

${ }^{1}$ Major in Mathematics, College of Natural Science, Keimyung University, Daegu, South Korea. ${ }^{2}$ Department of Mathematics Education, Pusan National University, Busan, South Korea.

\section{Publisher's Note}

Springer Nature remains neutral with regard to jurisdictional claims in published maps and institutional affiliations.

Received: 22 April 2018 Accepted: 28 September 2018 Published online: 04 October 2018

\section{References}

1. Cabada, A., Dimitrijevic, S., Tomovic, T., Aleksic, S.: The existence of a positive solution for nonlinear fractional differential equations with integral boundary value conditions. Math. Methods Appl. Sci. 40(6), 1880-1891 (2017)

2. Cui, Y., Sun, J.: On existence of positive solutions of coupled integral boundary value problems for a nonlinear singular superlinear differential system. Electron. J. Qual. Theory Differ. Equ. 2012, 41 (2012)

3. Cui, Y., Zou, Y.: Monotone iterative method for differential systems with coupled integral boundary value problems. Bound. Value Probl. 2013, 245 (2013)

4. Cui, Y., Zou, Y.: An existence and uniqueness theorem for a second order nonlinear system with coupled integral boundary value conditions. Appl. Math. Comput. 256, 438-444 (2015)

5. Feng, M., Ji, D., Ge, W.: Positive solutions for a class of boundary-value problem with integral boundary conditions in Banach spaces. J. Comput. Appl. Math. 222(2), 351-363 (2008)

6. Ghergu, M., Radulescu, V.: Nonlinear PDEs. Mathematical Models in Biology, Chemistry and Population Genetics. Springer Monographs in Mathematics. Springer, Heidelberg (2012)

7. Guo, D., Lakshmikantham, V.: Nonlinear Problems in Abstract Cones. Academic Press, Orlando (1988)

8. Kong, Q., George, T.S.: Linear Sturm-Liouville problems with Riemann-Stieltjes integral boundary conditions. Opusc. Math. 38(4), 557-571 (2018)

9. Lee, Y.H.: Multiplicity of positive radial solutions for multiparameter semilinear elliptic systems on an annulus. J. Differ. Equ. 174(2), 420-441 (2001)

10. Liu, X., Jia, M.: Existence of solutions for the integral boundary value problems of fractional order impulsive differential equations. Math. Methods Appl. Sci. 39(3), 475-487 (2016)

11. Webb, J.R.L., Infante, G.: Positive solutions of nonlocal boundary value problems: a unified approach. J. Lond. Math. Soc. (2) 74(3), 673-693 (2006)

12. Yan, B.: Positive solutions for the singular nonlocal boundary value problems involving nonlinear integral conditions. Bound. Value Probl. 2014, 38 (2014)

13. Yang, Z.: Positive solutions to a system of second-order nonlocal boundary value problems. Nonlinear Anal. 62(7), 1251-1265 (2005)

\section{Submit your manuscript to a SpringerOpen ${ }^{\circ}$ journal and benefit from:}

- Convenient online submission

- Rigorous peer review

- Open access: articles freely available online

- High visibility within the field

- Retaining the copyright to your article 